\title{
Masa intramiocárdica asintomática en un adulto mayor
}

\author{
Silvia Makhoul*, \\ Esteban L Clos** \\ Cynthia Kudrle Cozzolino***
}

Correspondencia

Silvia makhoul

silviamakhoul@hotmail.com

* Servicio de cardiología Hospital Británico de Buenos Aires. Hospital Juan A Fernández. Argentina

** Servicio de cardiología Hospital Británico de Buenos Aires. Departamento de cardiología Investigaciones Médicas. Argentina

*** Servicio de cardiología Hospital Británico de Buenos. Argentina

Recibido: 24/8/2020

Aceptado: $23 / 02 / 2021$

En línea: 30/04/2021

Citar como: Makhoul S, Clos E, Kurdle Cozzolino C. Masa intramiocárdica asintomática en un adulto mayor. Rev Ecocar Pract (RETIC). 2021 (Abril); 4 (1): 1-3. doi: 10.37615/retic.v4n1a3.

Cite this as: Makhoul S, Clos E, Kurdle Cozzolino C. Asymptomatic intramyocardial mass in an older patient. Rev Ecocar Pract (RETIC). 2021 (Abril); 4 (1): 1-3. doi: 10.37615/retic.v4n1a3.

\section{Palabras clave \\ $\triangleright$ Masas cardíacas \\ $\triangleright$ Ecocardiografía \\ $\triangleright$ Resonancia cardíaca}

\begin{tabular}{l}
\hline Keywords \\
\hline$\triangleright$ Cardiac masses \\
$\triangleright$ Echocardiography \\
$\triangleright$ Cardiac resonance \\
\end{tabular}

Presentación del caso

Paciente varón de 79 años que consultó por el hallazgo incidental de una masa intramiocárdica en un chequeo general de salud. Él se encontraba asintomático, jugaba al golf 3 veces por semana, no tenía factores de riesgo mayores, ni antecedentes patológicos relevantes.

Su examen físico, análisis de laboratorio (eritrosedimentación-VSG: 4mm) y electrocardiograma eran normales. En dicha evaluación general se le solicitó un ecocardiograma y una prueba de perfusión miocárdica (PPM). La PPM No evidenció isquemia, su capacidad funcional fue de 12 Mets y se informó una secuela inferior. En el ecocardiograma se observó una masa intramiocárdica, redondeada en la pared posterior del ventrículo izquierdo $(\mathrm{VI})$, hiperrefringente, con bordes definidos (Videos 1, 2 y Figuras 1, 2) de 1,8 2 2,6 cm, que no generaba un gradiente significativo intraventricular. Las cavidades cardíacas eran normales, la función sistólica del VI fue normal y no presentaba derrame pericárdico. Por tal motivo se solicitó una resonancia magnética cardíaca con contraste (Videos 3, 4) para caracterizar dicha masa.

Debido a su localización, forma y tamaño, inicialmente los diagnósticos presuntivos fueron fibroma, angiofibroma, hemangioma o metástasis.
La resonancia detectó a nivel del segmento medio inferior del VI una masa intramiocárdica redondeada, de $22 \times 21 \mathrm{~mm}$, de bordes bien definidos, sin invasión a estructuras vecinas ni derrame pericárdico. La misma fue levemente hipointensa a isointensa en $\mathrm{T} 1$ y $\mathrm{T} 1$ con supresión grasa e isointensa a hiperintensa en secuencias de cine y significativamente hiperintensa en T2 con supresión grasa. Durante la infusión de gadolinio, se observó intensa perfusión de la misma, con intenso realce tardío a los 10 minutos. (Videos 3,4 y Figura 3).

Este informe puso en evidencia una masa de bordes definidos, sin invasión del pericardio, con escaso contenido graso y vascularizada.

Los algoritmos diagnósticos en base a los hallazgos de la resonancia cardíaca nos llevaron a la sospecha diagnóstica de un probable hemangioma, el cual sólo podrá ser confirmado por el análisis histopatológico.

El paciente no presentaba masas en otras regiones de su organismo. El registro del Holter electrocardiográfico de 24 horas fue normal.

En imágenes de estudios realizados en el paciente en los últimos 6 años (en ellos la masa no fue jerarquizada), se observa la masa de iguales características, sin progresión en el tiempo. 
En este tipo de tumores, benignos e irresecables, en pacientes asintomáticos, se recomienda el tratamiento conservador. No obstante, el paciente realizó consultas con cirujanos cardiovasculares quienes consideraron a la masa intramiocárdica "irresecable y de difícil abordaje para realizar una biopsia".

En este caso el paciente lleva 10 años asintomático y sin observarse modificaciones evolutivas de la masa intramiocárdica en las diferentes modalidades de imágenes.

Es de destacar que el abordaje inicial del tipo de tumor se hizo a través del ecocardiograma, pero fue la resonancia magnética, a través de la caracterización tisular, la que permitió aproximarnos más a las características histológicas de tumor. Sin embargo, sólo la anatomía patológica definirá el caso.

\section{Estudio por imagen}

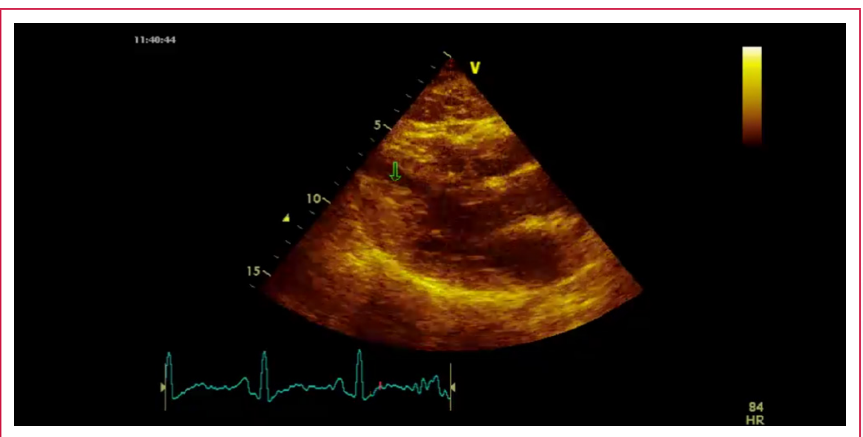

Vídeo 1. Ecocardiograma transtorácico: paraesternal eje largo, masa protruyente en pared posterior del ventrículo izquierdo

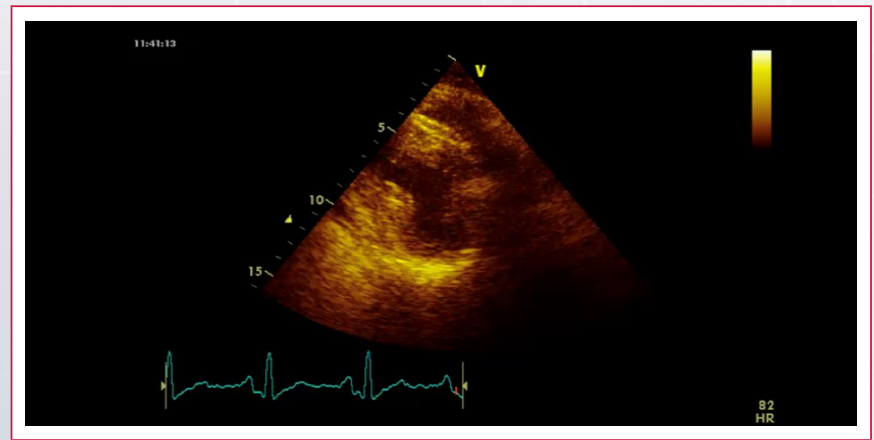

Vídeo 2. Ecocardiograma transtorácico: paraesternal eje corto, masa protruyente en pared posterior del VI

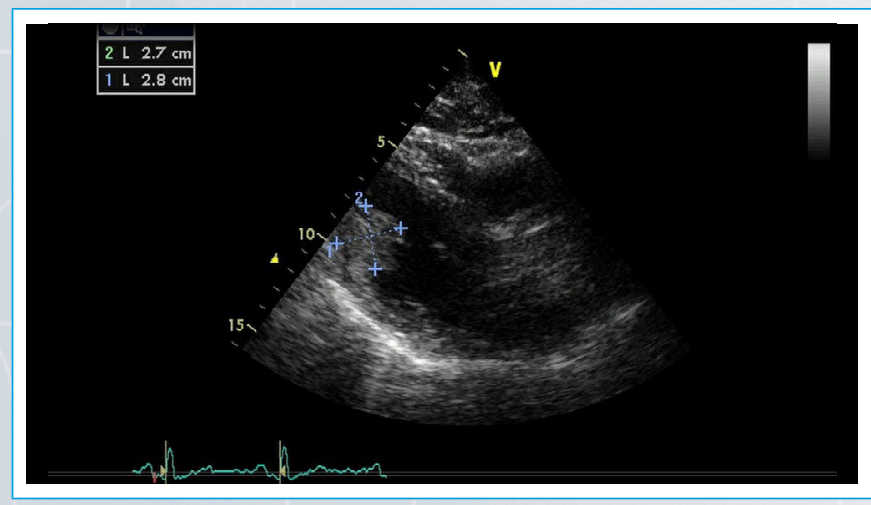

Figura 1. Ecocardiograma transtorácico: paraesternal eje largo, con masa protruyente en pared posterior del ventrículo izquierdo (VI)

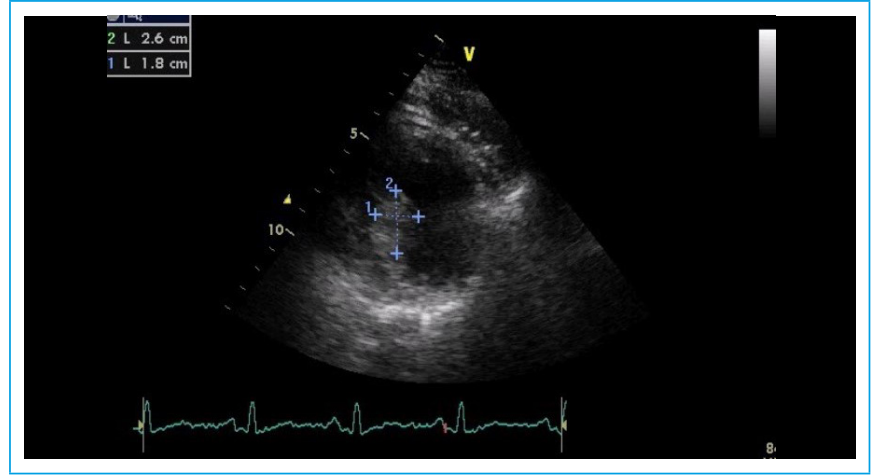

Figura 2. Ecocardiograma transtorácico: paraesternal eje corto, con masa protruyente en pared posterior del VI

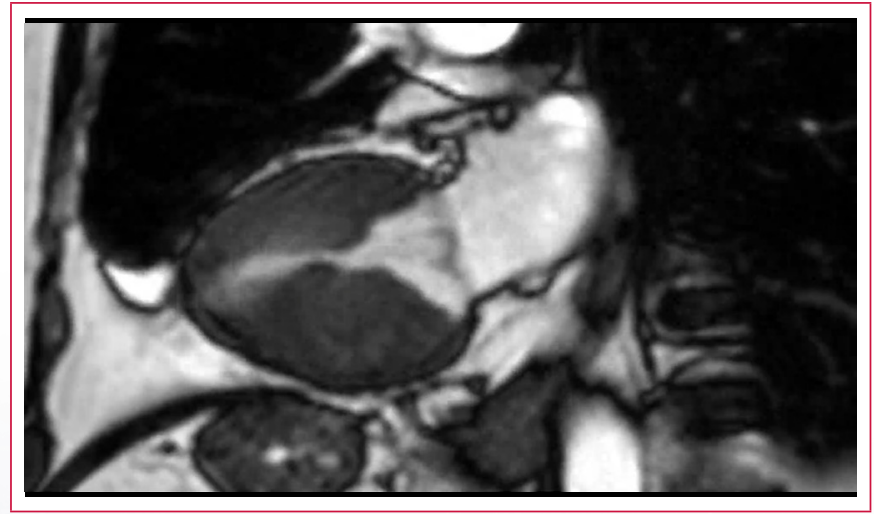

Vídeo 3. Resonancia magnética cardíaca (RMC): apical 2 cámaras, masa isointensa en pared posterior, estudio basal

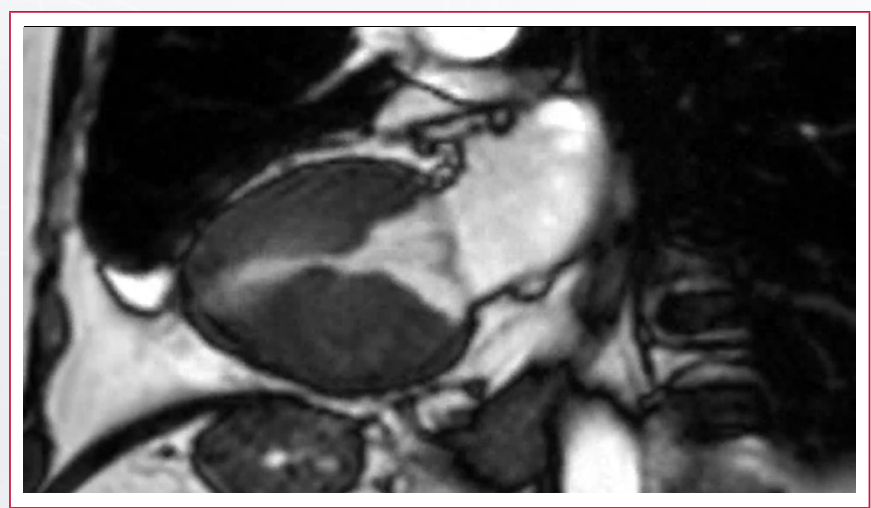

Vídeo 4. Resonancia magnética cardíaca (RMC): apical 2 cámaras, perfusión de la masa post gadolinio

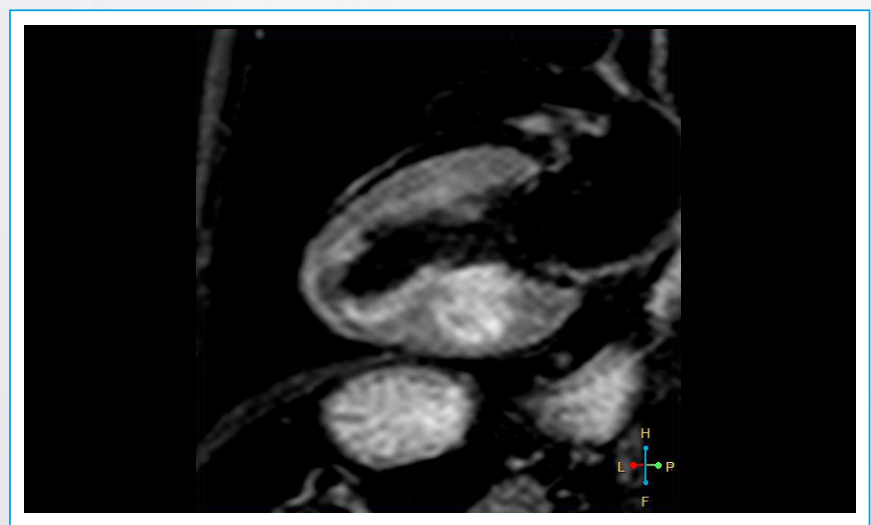

Figura 3. Resonancia magnética cardíaca (RMC): apical 2 cámaras, secuencia potenciada en T2 con supresión grasa 


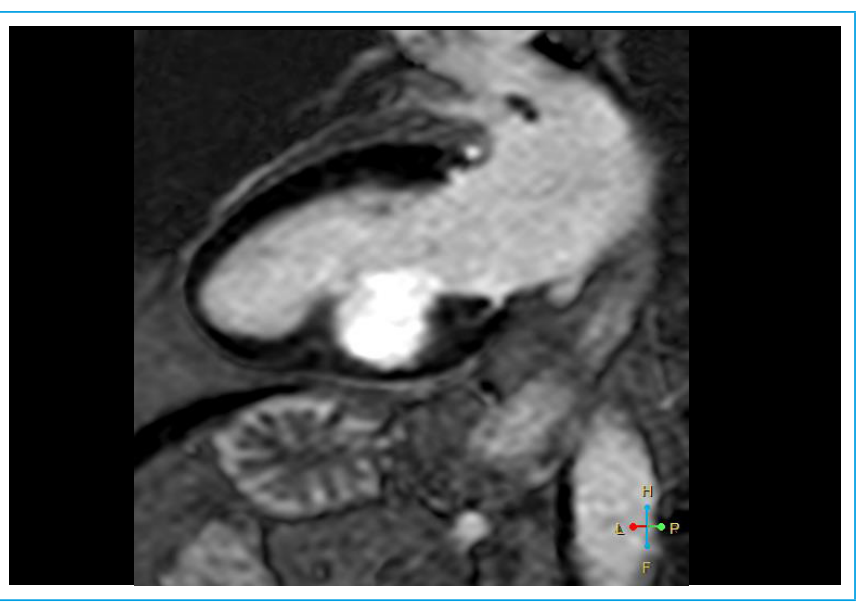

Figura 4. Resonancia magnética cardíaca (RMC): apical 2 cámaras, captación de gadolinio tardía por la masa

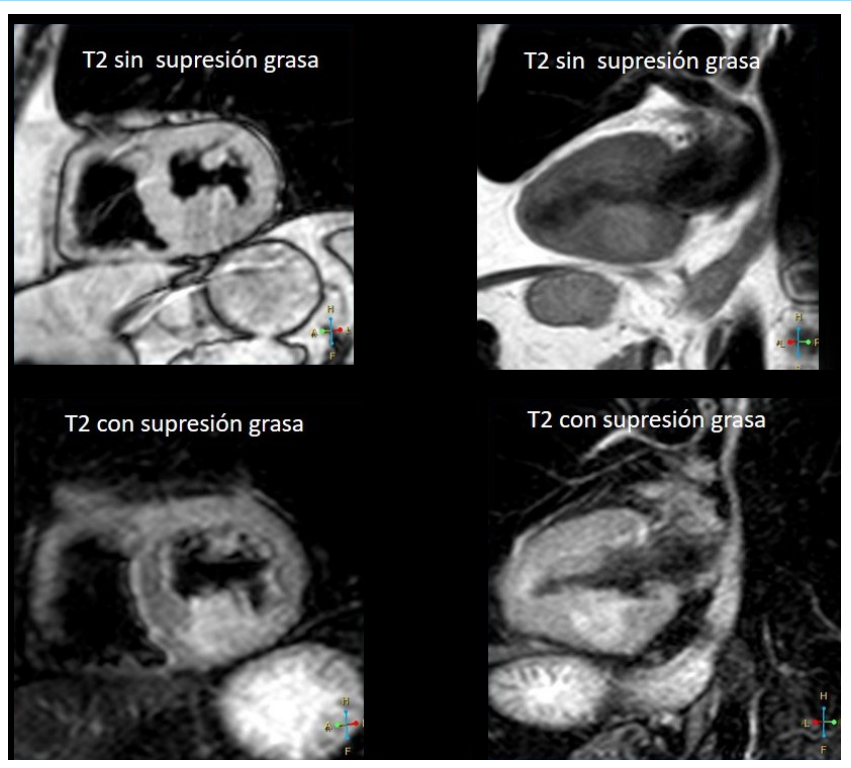

Figura 5. Resonancia magnética cardíaca (RMC): apical 2 cámaras y eje corto en secuencias potenciadas en T2 con y sin supresión grasa

\section{Discusión}

Los tumores cardíacos tienen una presentación clínica muy variable y escasamente orientativa hacia un diagnóstico certero, por lo que constituye un verdadero desafío su detección, diagnóstico etiológico y tratamiento.

Las metástasis cardíacas son más frecuentes que los tumores cardíacos primarios, siendo los tumores benignos los de mayor incidencia. Estos pueden tener diferentes localizaciones: intracavitarios, intramiocárdicos e intrapericárdicos.

Los tumores cardíacos primarios son raros, su incidencia oscila entre el 0,02$0,056 \%$. De ellos, el mixoma es el tumor benigno más frecuente, representando el $50 \%$ de los mismos, mientras que los hemangiomas constituyen el 5-10\% de todos los tumores primarios benignos y son tumores vasculares con proliferación de células endoteliales ${ }^{(1-3)}$.

Los hemangiomas intramurales se localizan con mayor frecuencia en el ventrículo derecho e izquierdo, aunque también se reportaron hemangiomas en aurícula izquierda (Al), orejuela de Al, aurícula derecha e incluso en el pericardio. ${ }^{(7.8)}$. La resonancia cardíaca nos aproxima al diagnóstico ante la presencia de masas cardíacas con imágenes que evidencian las siguientes características: señal de densidad intermedia en T1, hiperintensas en las secuencias de T2 y realce tardío del gadolinio ${ }^{(1,3,7,8)}$.

El diagnóstico pre-operatorio es posible sólo en un tercio de los pacientes y la evolución de los mimos es variable: algunos proliferan, otros no modifican su crecimiento en el tiempo e incluso pueden involucionar. Por ello es fundamental el seguimiento a través de las imágenes cardiovasculares ${ }^{(4-7,8)}$.

Quizá el mayor desafío sea tomar una conducta adecuada, particularmente cuando el tumor es un hallazgo incidental y el paciente se encuentra asintomático.

\section{Conclusiones}

La evaluación de masas cardíacas es un desafío para las diferentes técnicas de imagen cardiovascular. El abordaje inicial, debe realizarse a través de la ecocardiografía y complementar su estudio a través de otras modalidades como la resonancia magnética cardíaca; que evalúa con mayor precisión las características de la masa (tamaño, morfología, localización, perfusión) y su probabilidad de malignidad, a través de las diferentes secuencias de mapeo y el uso de contraste.

\section{Ideas para recordar}

- Las metástasis son los tumores cardíacos más frecuentes.

- Los tumores benignos primarios cardíacos son raros, siendo el hemangioma uno de los menos frecuentes.

- La resonancia cardíaca nos permite realizar la caracterización tisular y descartar signos de malignidad de las masas cardíaca.

\section{Bibliografía}

1. García Fernández MA, Pérez de la Isla L, Gómez de Diego JJ, Macaya Miguel C. Tratado de Cardiología Clínica. Oncocardiologia. Cap 49. Madrid. CTO Editorial SL 2015. Grupo CTO

2. Gomez de Diego JJ, Garcia Fernández MA, Lancellotti P, Garbi M, Perez de Isla L. Hernández Vyhmeister Ry col. Recomendamos la lectura del "Atlas de imagen cardiaca". Cap 13. Masas cardiacas: 475-522. Ed Grupo CTO

3. Arisha MA, Hsiung MC, Nanda NC et al. Incremental benefit of three-dimensional Transesophageal echocardiography in the assessment of a primary pericardial hemangioma. Echocardiography. 2017;1-6.

4. Tyebally S,Chen D,Bhattacharyya S et al. Cardiac Tumors. J Am Coll Cardiol CardioOnc 2020;2:293-311.

5. Soberman MS, Plauth WH, Winn KJ y col. Hemangioma of the right ventricle causing outflow tract obstrucction. J Thorac Cardiovasc Surg 1988; 96:307-309.

6. Kann BR, Kim WJ, Cilley JH y col. Hemangioma of the right ventricular outflow tract. Ann Thorac Surg 2000; 70:975-977.

7. Manasse E, Nicolini F, Canziani R y col. Left ventricular hemangioma. Eur J Cardiothorac Surg 1999; 15:864-866.

8. Markarian L y col. Hemangiomas cardiacos: revisión y actualización. Claves de Cardiologia 2007;2 (7):1-4.

9. Celya AC, Jiménez López-Guarchc C y Charterinae S. Masas cardiacas: multimodalidad-resonancia magnética cardiaca. Rev Colomb Cardiol. 2019;26(S1):111-122. 